\title{
Precise determination of the low-energy hadronic contribution to the muon $g-2$ from analyticity and unitarity: An improved analysis
}

\author{
B.Ananthanarayan, ${ }^{1}$ Irinel Caprini, ${ }^{2}$ Diganta Das, ${ }^{3}$ and I. Sentitemsu Imsong ${ }^{\dagger 4}$ \\ ${ }^{1}$ Centre for High Energy Physics, Indian Institute of Science, Bangalore 560 012, India \\ ${ }^{2}$ Horia Hulubei National Institute for Physics and Nuclear Engineering, P.O.B. MG-6, 07r125 Magurele, Romania \\ ${ }^{3}$ Physical Research Laboratory, Navrangpura, Ahmedabad 380 009, India \\ ${ }^{4}$ The Institute of Mathematical Sciences, Taramani, Chennai 600 113, India
}

\begin{abstract}
The two-pion low-energy contribution to the anomalous magnetic moment of the muon, $a_{\mu} \equiv$ $(g-2)_{\mu} / 2$, expressed as an integral over the modulus squared of the pion electromagnetic form factor, brings a relatively large contribution to the theoretical error, since the low accuracy of experimental measurements in this region is amplified by the drastic increase of the integration kernel. We derive stringent constraints on the two-pion contribution by exploiting analyticity and unitarity of the pion electromagnetic form factor. To avoid the poor knowledge of the modulus of this function, we use instead its phase, known with high precision in the elastic region from Roy equations for pion-pion scattering via the Fermi-Watson theorem. Above the inelastic threshold we adopt a conservative integral condition on the modulus, determined from data and perturbative QCD. Additional high precision data on the modulus in the range $0.65-0.71 \mathrm{GeV}$, obtained from $e^{+} e^{-}$annihilation and $\tau$-decay experiments, are used to improve the predictions on the modulus at lower energies by means of a parametrization-free analytic extrapolation. The results are optimal for a given input and do not depend on the unknown phase of the form factor above the inelastic threshold. The present work improves a previous analysis based on the same technique, including more experimental data and employing better statistical tools for their treatment. We obtain for the contribution to $a_{\mu}$ from below $0.63 \mathrm{GeV}$ the value $(133.258 \pm 0.723) \times 10^{-10}$, which amounts to a reduction of the theoretical error by about $6 \times 10^{-11}$.
\end{abstract}

PACS numbers: 11.55.Fv, 13.40.Gp, 25.80.Dj

\section{INTRODUCTION}

The muon anomalous magnetic moment is one of the most precisely measured observables in particle physics. It can also be predicted by theory with a high accuracy, and is therefore an ideal quantity for testing the standard model and for finding possible deviations from it caused by new physics [1, 2]. The great interest in muon anomaly is motivated by the present discrepancy of about 3 to 4 $\sigma$ between theory and experiment. For recent reviews, see Refs. [3 6] (see also the bibliography in e.g, 7]). New generation measurements of muon $g-2$ planned at Fermilab [8] and JPARC [9] are expected to produce results with experimental errors at the level of $16 \times 10^{-11}$, a factor of 4 smaller compared to the Brookhaven measurement [10]. This therefore requires a precision at the same level also for the theoretical result.

The largest theoretical uncertainties are related to the hadronic contribution to $a_{\mu}$, which comes mainly from energies at which the confined quarks are strongly interacting and the QCD perturbative treatment breaks down. The evaluation of the nonperturbative effects is usually done by means of dispersion relations in conjunction with experimental data. Low energy effective theo-

\footnotetext{
${ }^{\dagger}$ Deceased.
}

ries and lattice QCD are also used. Efforts are currently made to increase the precision of these calculations, regarding both the hadronic vacuum polarization and the hadronic light-by-light scattering (a compilation of recent studies is presented in [11]).

The hadronic vacuum polarization (VP), which is numerically the most significant term, contributes with about $43 \times 10^{-11}$ units to the theoretical error. It is dominated by the two-pion contribution, which brings more than $70 \%$ of the leading-order hadronic contribution.

The two-pion contribution to the VP is expressed in terms of the modulus squared of the pion electromagnetic form factor. It has been measured in $e^{+} e^{-}$annihilation experiments by CMD2 [12 14], SND [15], $B A B A R$ [16, 17], KLOE [18 20] and BESIII [21], and from the hadronic decays of the $\tau$ lepton by CLEO [22], ALEPH [23, 24], OPAL [25] and Belle [26]. Due to experimental difficulties in the identification of low-energy pions, the data below $0.6 \mathrm{GeV}$ have very large uncertainties, except for $B A B A R$ and KLOE. The recent data published by BESIII [21] are restricted to energies above 0.6 $\mathrm{GeV}$. Two new detectors, CMD-3 and SND, now operating at the VEPP-2000 $e^{+} e^{-}$collider in Novosibirsk, are expected to bring accurate data of greatest interest for the $a_{u}$ evaluation 27 29]. Preliminary results reported in [30, 31] indicate as goal an accuracy comparable to that of $B A B A R$ and KLOE experiments.

The lack of data of sufficient precision at low energies, combined with the fact that the integration kernel 
exhibits a drastic increase in this region, leads to a relatively large uncertainty of the corresponding contribution to the muon anomaly 32 34. The contribution to $a_{\mu}$ from below $0.63 \mathrm{GeV}$, obtained using a fit of the pion form factor in the region near threshold and the direct integration of a compilation of data on $e^{+} e^{-} \rightarrow \pi^{+} \pi^{-}$ cross section between 0.30 and $0.63 \mathrm{GeV}$, is quoted in 32 with an error of $13.1 \times 10^{-11}$, while the direct integration in this range of the $B A B A R$ data alone leads to an error of $14.7 \times 10^{-11}$.

The present large uncertainty of the two-pion contribution to $a_{\mu}$ from energies below $0.63 \mathrm{GeV}$ motivated us recently 35] to investigate it theoretically in a framework based on the analyticity and unitarity properties of the pion form factor. The main idea was to use, instead of the poorly known modulus, the phase of the form factor, which is equal by the Fermi-Watson theorem [36, 37] to the $\pi \pi$ scattering $P$-wave phase shift, which has been calculated with high precision from Chiral Perturbation Theory (ChPT) and Roy equations 38 40. Above the inelastic threshold, where the Fermi-Watson theorem is no longer valid and the phase of the form factor is not known, we have used an integral condition on the form-factor modulus, derived using measurements of the $B A B A R$ experiment [16, 17] up to $3 \mathrm{GeV}$ and the asymptotic behavior of the form factor predicted by perturbative QCD [41 43] above that energy.

The knowledge of the phase on a part of the unitarity cut and of the modulus on the other part of the cut is not sufficient for uniquely predicting the form factor. However, as shown first in [4], from this information one can derive rigorous upper and lower bounds on the modulus below the inelastic threshold, in particular in the low energy region. To increase the strength of the bounds, we have used as input also several values of the modulus from the region $0.65-0.71 \mathrm{GeV}$, measured with higher precision by the $e^{+} e^{-}$experiments CMD2 [12], SND [15] $B A B A R$ [16, 17] and KLOE 13 [20]. The method amounts to a parametrization-free analytic extrapolation from higher energies to the low energy region of interest for the improved calculation of the muon anomaly. It led to a two-pion contribution to $a_{\mu}$ from the region below $0.63 \mathrm{GeV}$ which agreed with other re- cent determinations and had a smaller uncertainty 35].

In the present paper we present an update of the work [35], improving certain details of the analysis. The main improvement is a proper treatment by Monte Carlo simulations of the statistical errors of the data used as input, which will allow us to attach an uncertainty to the result at a precise confidence level (C.L.). Also, better tools [45] for combining different predictions accounting for their possible correlations are used. In addition to the $e^{+} e^{-}$ data from the region $0.65-0.71 \mathrm{GeV}$ used as input in 35], we also consider the KLOE independent measurements reported in [19] and the very recent data of BESIII [21]. We include also the data obtained in the same energy region from $\tau$-lepton decays by the CLEO 22], ALEPH [23, 24], OPAL 25] and Belle [26] collaborations.

The outline of this paper is as follows: in Sec. II we formulate our aim and review the conditions used as input. In Sec. III we give a detailed description of the experimental information used as input and in Sec. IV we describe the Monte Carlo simulation used for implementing the statistical uncertainties of the input data and the prescription of combining the predictions from different experiments. Section $\nabla$ contains our results and Sec. VI a summary and our conclusions. The paper has two Appendices: in Appendix $\mathrm{A}$ we present the solution of the functional extremal problem formulated in Sec. III which is the mathematical basis of our approach. In Appendix B we discuss the extraction of the pion form factor from the $e^{+} e^{-}$and $\tau$-decay experiments, giving a short overview of various corrections applied.

\section{FORMALISM}

We consider the leading order (LO) two-pion contribution to $a_{\mu}$, which does not contain the vacuum polarization effects but includes one-photon final-state radiation (FSR). We are interested in finding the two-pion contribution to $a_{\mu}$ from the interval of energies ranging from $\sqrt{t_{\text {low }}}$ to $\sqrt{t_{\text {up }}}$, which is expressed in terms of the pion electromagnetic form factor $F(t)$ as

$$
a_{\mu}^{\pi \pi(\gamma), \mathrm{LO}}\left[\sqrt{t_{\mathrm{low}}}, \sqrt{t_{\mathrm{up}}}\right]=\frac{\alpha^{2} m_{\mu}^{2}}{12 \pi^{2}} \int_{t_{\mathrm{low}}}^{t_{\mathrm{up}}} \frac{d t}{t} K(t) \beta_{\pi}^{3}(t)|F(t)|^{2}\left|F_{\omega}(t)\right|^{2}\left(1+\frac{\alpha}{\pi} \eta_{\pi}(t)\right)
$$

In this relation, $\beta_{\pi}(t)=\left(1-4 m_{\pi} / t\right)^{1 / 2}$ is the two-pion phase space relevant for $e^{+} e^{-} \rightarrow \pi^{+} \pi^{-}$annihilation $\left(m_{\pi}\right.$ being the charged pion mass), and

$$
K(t)=\int_{0}^{1} d u(1-u) u^{2}\left(t-u+m_{\mu}^{2} u^{2}\right)^{-1}
$$

is the QED kernel function. This function is known to exhibit a drastic increase at low $t[1]$.

The integrand in (11) contains the pion electromagnetic form factor $F(t)$ in the isospin limit, defined by

$$
\left\langle\pi^{+}\left(p^{\prime}\right)\left|J_{\mu}^{\mathrm{elm}}\right| \pi^{+}(p)\right\rangle=\left(p+p^{\prime}\right)_{\mu} F(t), t=\left(p-p^{\prime}\right)^{2},
$$


which is a real analytic function in the $t$ complex plane cut along the real semiaxis $t \geq 4 m_{\pi}^{2}$. The remaining factors in (1) denote corrections not included in the form factor: $F_{\omega}(t)$ accounts for the isospin violation due to $\rho-\omega$ mixing and is parametrized as [46, 47]]:

$$
F_{\omega}(t)=1+\epsilon \frac{t}{\left(m_{\omega}-i \Gamma_{\omega} / 2\right)^{2}-t},
$$

where $\epsilon=1.9 \times 10^{-3}$. Finally, $\eta_{\pi}(t)$ is the FSR correction, calculated in scalar QED [48, 49].

We are interested in the contribution to (10) of the energies below $0.63 \mathrm{GeV}$. For convenience we shall use in what follows the simplified notation

$$
a_{\mu} \equiv a_{\mu}^{\pi \pi(\gamma), \mathrm{LO}}\left[2 m_{\pi}, 0.63 \mathrm{GeV}\right]
$$

We now formulate the conditions on the form factor $F(t)$ adopted as input for constraining the above quantity. Following Ref. [35], we write these conditions as:

1. Fermi-Watson theorem [36, 37], which implies:

$$
\operatorname{Arg}[F(t+i \epsilon)]=\delta_{1}^{1}(t), \quad 4 m_{\pi}^{2} \leq t \leq t_{\text {in }},
$$

where $\delta_{1}^{1}(t)$ is the phase-shift of the $P$-wave of $\pi \pi$ elastic scattering and $t_{\text {in }}$ is the first inelastic threshold.

2. Normalization at $t=0$ and the value of the charge radius $\left\langle r_{\pi}^{2}\right\rangle$, expressed by:

$$
F(0)=1, \quad\left[\frac{d F(t)}{d t}\right]_{t=0}=\frac{1}{6}\left\langle r_{\pi}^{2}\right\rangle .
$$

3. An integral condition on the modulus squared above the inelastic threshold, written in the form

$$
\frac{1}{\pi} \int_{t_{\mathrm{in}}}^{\infty} d t \rho(t)|F(t)|^{2} \leq I,
$$

where $\rho(t)$ is a suitable positive-definite weight, for which the integral converges and an accurate evaluation of $I$ is possible.

4. The value at one spacelike energy, known from experiment:

$$
F\left(t_{s}\right)=F_{s} \pm \epsilon_{s}, \quad t_{s}<0 .
$$

5. The modulus at one energy in the elastic region of the timelike axis, known from experiment:

$$
\left|F\left(t_{t}\right)\right|=F_{t} \pm \epsilon_{t}, \quad 4 m_{\pi}^{2}<t_{t}<t_{\text {in }}
$$

As in Ref. [35], we consider the following functional extremal problem: using as input the conditions 1-5, we derive optimal upper and lower bounds on $|F(t)|$ at all points on the elastic unitarity cut, $4 m_{\pi}^{2}<t<t_{\text {in }}$, in particular at energies below $0.63 \mathrm{GeV}$ of interest for the calculation of the quantity (5). The solution of the extremal problem and the algorithm for obtaining the bounds are presented for completeness in Appendix A. In order to operate this machinery, we need high quality phenomenological inputs, which are the subject of the following section.

\section{PHENOMENOLOGICAL INPUT}

In this section we briefly describe the input used in the conditions 1-5, expressed in the Eqs. (6)-(10) given in the previous section.

The first significant inelastic threshold $t_{\text {in }}$ for the pion form factor is due to the opening of the $\omega \pi$ channel, i.e. $\sqrt{t_{\text {in }}}=m_{\omega}+m_{\pi}=0.917 \mathrm{GeV}$. Below this threshold, we use in (6) the phase shift $\delta_{1}^{1}(t)$ from Refs. [38, 39] and [40], which we denote as Bern and Madrid phase, respectively.

For the charge radius entering (77) we use the constraint $\left\langle r_{\pi}^{2}\right\rangle \in(0.41,0.45) \mathrm{fm}^{2}$ derived in [50]. Since this range was obtained basically from the same constraints as those listed in the previous section, the knowledge of the charge radius plays actually a weak role in further improving the bounds on the modulus in the energy region of interest. However, we keep this condition since we now use a different treatment of the uncertainties compared to our previous analyses.

We have calculated the integral (8) using the $B A B A R$ data [16] from $t_{\text {in }}$ up to $\sqrt{t}=3 \mathrm{GeV}$, smoothly continued with a constant value for the modulus in the range $3 \mathrm{GeV} \leq \sqrt{t} \leq 20 \mathrm{GeV}$, and a $1 / t$ decreasing modulus at higher energies, as predicted by perturbative QCD [4143]. As discussed in detail in Refs. 35, 51, 52 this evaluation is expected to overestimate the true value of the integral. As in 35] we have adopted the weight $\rho(t)=1 / t$, for which the contribution of the range above $3 \mathrm{GeV}$ to the integral (8) is only of $1 \%$. The value of $I$ obtained with this weight is 35

$$
I=0.578 \pm 0.022,
$$

where the uncertainty is due to the $B A B A R$ experimental errors. In the calculations we have used as input for $I$ the central value quoted in Eq. (11) increased by the error, which leads to the most conservative bounds due to a monotonicity property discussed in Appendix A.

For the spacelike input (9) we have used the most recent experimental determinations [53, 54]

$$
\begin{aligned}
F\left(-1.60 \mathrm{GeV}^{2}\right) & =0.243 \pm 0.012_{-0.008}^{+0.019} \\
F\left(-2.45 \mathrm{GeV}^{2}\right) & =0.167 \pm 0.010_{-0.007}^{+0.013}
\end{aligned}
$$

As shown in [35], a major role in increasing the strength of the bounds is played by condition (10), with $0.65 \mathrm{GeV} \leq \sqrt{t_{t}} \leq 0.71 \mathrm{GeV}$. This energy region was chosen since it is close to the region of interest and therefore has a stronger effect on improving the bounds than the input from higher energies. The $e^{+} e^{-}$data are taken below $0.705 \mathrm{GeV}$ and the $\tau$-decay data below $0.710 \mathrm{GeV}$, with the exception of one datum from CLEO that corresponds to an energy of $0.712 \mathrm{GeV}$. Since this last datum is at an energy that is only marginally higher than the upper limit of the aforementioned energy range, it is included in the analysis. It is noteworthy that in this region the modulus measured by various experiments exhibits smaller variations than in other energy regions and a higher degree of mutual consistency. 

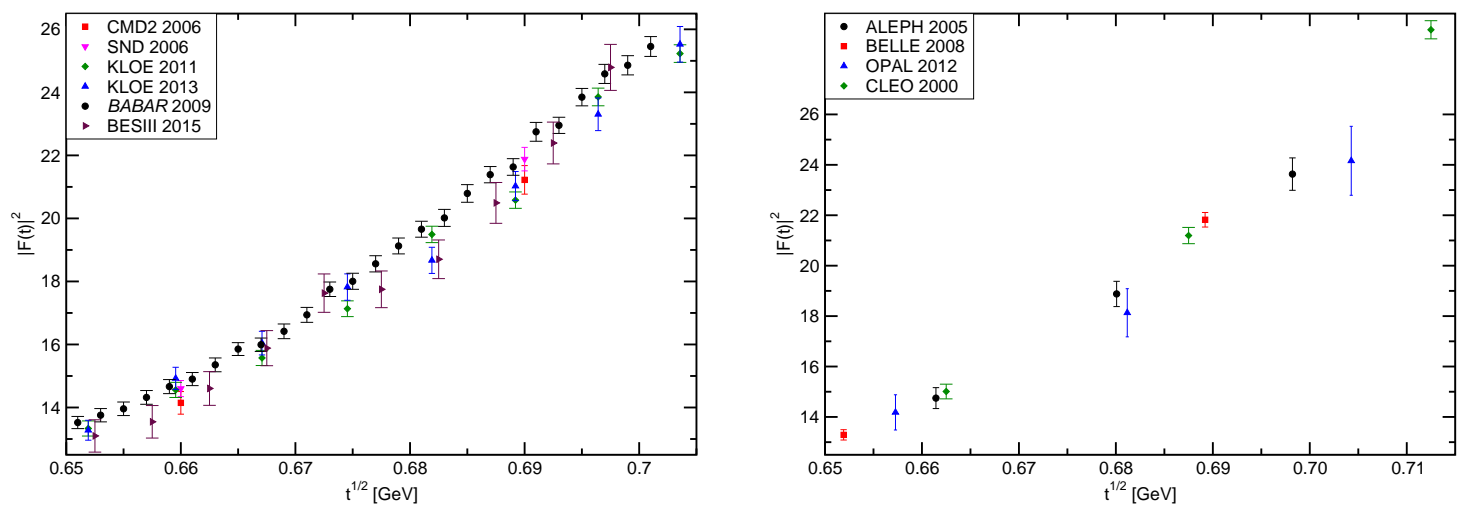

FIG. 1: Modulus squared $|F(t)|^{2}$ measured in the region $0.65-0.71 \mathrm{GeV}$ by $e^{+} e^{-}$-annihilation (left) and $\tau$-decay (right) experiments.

\begin{tabular}{lr}
\hline Experiment & Number of points \\
\hline CMD2 [12] & 2 \\
SND [15] & 2 \\
BABAR [16, 17] & 26 \\
KLOE 2011 [19] & 8 \\
KLOE 2013 [20] & 8 \\
BESIII [21] & 10 \\
\hline CLEO [22] & 3 \\
ALEPH [23, 24] & 3 \\
OPAL [25] & 3 \\
Belle [26] & 2 \\
\hline
\end{tabular}

TABLE I: Number of points in the region $0.65 \mathrm{GeV} \leq \sqrt{t} \leq$ $0.71 \mathrm{GeV}$ where the modulus is measured by the $e^{+} e^{-}$annihilation and $\tau$-decay experiments considered in the analysis.

The numbers of experimental points in this range for various experiments, considered in our analysis, are summarized in Table [ We emphasize that in this region the $e^{+} e^{-}$-annihilation and $\tau$-decay experiments are fully consistent, so it is reasonable to use all the experiments on an equal footing. The extraction of the values of timelike modulus $|F(t)|$ from the cross-section of the process $e^{+} e^{-} \rightarrow \pi^{+} \pi^{-}$and the spectral function measured in $\tau$-decay experiments implies the application of several corrections, which ensure that the extracted quantity is indeed the form factor $F(t)$ defined in (3) in the isospin limit. Details are given in Appendix B Note that, for OPAL data we have used the rescaled values as presented in [55]. For completeness, we show in Fig. 1 the data on modulus squared $|F(t)|^{2}$ from the $e^{+} e^{-}$-annihilation and $\tau$-decay used as input in our analysis. It may be observed that the energies at which the form factor measurements are made vary from experiment to experiment. Therefore, it is not possible to combine the data and bring down the experimental error into the input itself.

\section{CALCULATION OF $a_{\mu}$ AND ITS UNCERTAINTY}

The algorithm presented in Appendix $\AA$ allows us to obtain rigorous bounds on $|F(t)|$ for $t$ in the region $\sqrt{t} \leq 0.63 \mathrm{GeV}$, in terms of the input specified in Sec. III We recall that the input consists of the phase of the form factor for $t \leq t_{\text {in }}$, the charge radius, one spacelike datum and one timelike modulus from the region $0.65-0.71 \mathrm{GeV}$. The lower and upper bounds (A16) are given by explicit expressions depending on definite values of the input. In practice, the input values are affected by uncertainties. In order to account for them, we have generated pseudorandom numbers for each of the input quantities with a priori given distributions. For the experimental spacelike and timelike data we have assumed Gaussian distributions with central value as the mean and the quoted errors as the standard deviation. For the spacelike data, symmetrized errors have been used in the Gaussian distributions, taking for symmetrization the biggest error [45]. The distributions of the phase and the charge radius, which are calculated from theoretical constraints, were assumed to be uniform.

For a timelike input in $0.65-0.71 \mathrm{GeV}$ region, a specified spacelike input and a selected phase (Bern or Madrid), a large sample $\left(\sim 10^{5}\right)$ of sets of inputs have been obtained by randomly drawing one value each from the above distributions. For each set of inputs in the sample, upper and lower bounds on the modulus squared $|F(t)|^{2}$ have been calculated using the algorithm of Appendix A, at each energy $\sqrt{t}$ from threshold to $0.63 \mathrm{GeV}$. All values in between the upper and lower bounds are equally probable. Therefore, for a given set of input (which yields one set of upper and lower bounds at each $\sqrt{t}$ ), at each low energy point $\sqrt{t}$ from threshold to 0.63 $\mathrm{GeV}$, a random admissible value between the bounds has been generated and used in the integration (11), yielding one value of the quantity $a_{\mu}$. The procedure is repeated 50 times for each set of inputs that yields 50 values of the 
quantity $a_{\mu}$. With this procedure, at each fixed timelike energy point, we obtain a large sample $\left(\sim 10^{6}\right)$ of the quantity $a_{\mu}$. The entire sample is binned to obtain a mean value and $68.3 \%$ confidence level upper and lower bounds at each timelike point.

The predictions obtained with input from different timelike energies were then combined into an average result for each experiment. The procedure of obtaining the average of several measurements in principle requires the knowledge of the correlations between the different values. Since these are not known ${ }^{1}$, we applied the averaging prescription proposed in [45], where the effective size of the correlations is estimated from the data themselves. As discussed in [45], the most robust average of a set of $n$ measurements $a_{i}$ is the weighted average

$$
\bar{a}=\sum_{i=1}^{n} w_{i} a_{i}, \quad w_{i}=\frac{1 / \delta a_{i}^{2}}{\sum_{j=1}^{n} 1 / \delta a_{j}^{2}},
$$

where $\delta a_{i}$ is the error of $a_{i}$.

For the best estimation of the error in the case of unknown correlations, the prescription proposed in [45] is to define a function $\chi^{2}(f)$

$$
\chi^{2}(f)=\sum_{i, j=1}^{n}\left(a_{i}-\bar{a}\right)\left(C(f)^{-1}\right)_{i j}\left(a_{j}-\bar{a}\right)
$$

in terms of the covariance matrix $C(f)$ with elements

$$
C_{i j}= \begin{cases}\delta a_{i} \delta a_{i} & \text { if } i=j \\ f \delta a_{i} \delta a_{j} & \text { if } i \neq j\end{cases}
$$

The parameter $f$ denotes the fraction of the maximum possible correlation: for $f=0$ the measurements are treated as uncorrelated, for $f=1$ as fully (100\%) correlated.

If $\chi^{2}(0)<n-1$, the data might indicate the existence of a positive correlation. The prescription proposed in [45] is to increase $f$ until $\chi^{2}(f)=n-1$. With the solution $f$ of this equation, the standard deviation $\sigma(\bar{a})$ of $\bar{a}$ is determined from the variance [45]

$$
\sigma^{2}(\bar{a})=\left(\sum_{i, j=1}^{n}\left(C(f)^{-1}\right)_{i j}\right)^{-1}
$$

On the other hand, if one obtains $\chi^{2}(0)>n-1$, this is an indication that the individual errors are underestimated. If the ratio $\chi^{2}(0) /(n-1)$ is not very far from 1 , the procedure suggested in [6, 45] is to rescale the variance $\sigma^{2}(\bar{a})$ calculated with (16) by the factor $\chi^{2}(0) /(n-1)$. In our

\footnotetext{
1 One can use as a first indication the bin-to-bin correlations of the input data on the modulus, which can be extracted in some cases from the published covariance matrices.
}

work, this kind of procedure was applied first for combining the results obtained with different measurements by each experiment. Then the predictions of various experiments were combined leading to a global average.

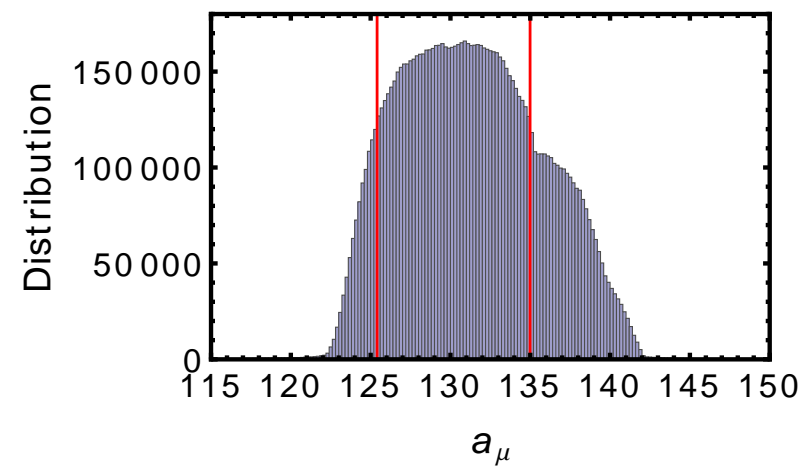

FIG. 2: Distribution of $a_{\mu}$ values obtained from the Monte Carlo sample of pseudodata, without input modulus. The vertical lines delimitate the region of $68.3 \%$ C.L.

\section{RESULTS}

It is instructive to first give the value obtained without using as input the measurements of the timelike modulus. In Fig. 2 we show the distribution of the $a_{\mu}$ sample, obtained using as input the Bern phase and the first spacelike point from (9). It may be readily seen that the distribution is not fully symmetrical, as it should be for a Gaussian distribution. From this distribution, by applying the $68.3 \%$ criterion we obtained for $a_{\mu}$ the value $\left(130.865_{-5.460}^{+4.124}\right) \times 10^{-10}$, and Madrid phase gives a similar result, $\left(131.933_{-5.922}^{+3.438}\right) \times 10^{-10}$. Since these results are not statistically independent, the most conservative procedure is to take the simple average of the central values and of the uncertainties. This gives

$$
a_{\mu}^{\pi \pi, \mathrm{LO}}\left[2 m_{\pi}, 0.63 \mathrm{GeV}\right]=\left(131.399_{-5.691}^{+3.780}\right) \times 10^{-10} .
$$

The large error shows that the constraining power of the phase and the spacelike data is rather low.

By including as input the modulus measured at one energy from the region $0.65-0.71 \mathrm{GeV}$, the determination (17) is considerably improved. In Fig. 3 we show for illustration the distribution of the $a_{\mu}$ sample, obtained using as input the Bern phase, the highest energy $B A B A R$ point shown in Fig. 1 and the first spacelike point from (9). The distribution is much narrower than that shown in Fig. 2 and more symmetrical, allowing the extraction of a smaller standard deviation by means of the $68.3 \%$ C.L. criterion.

Similar distributions of $a_{\mu}$ have been obtained for all the values of the input modulus measured in the region 


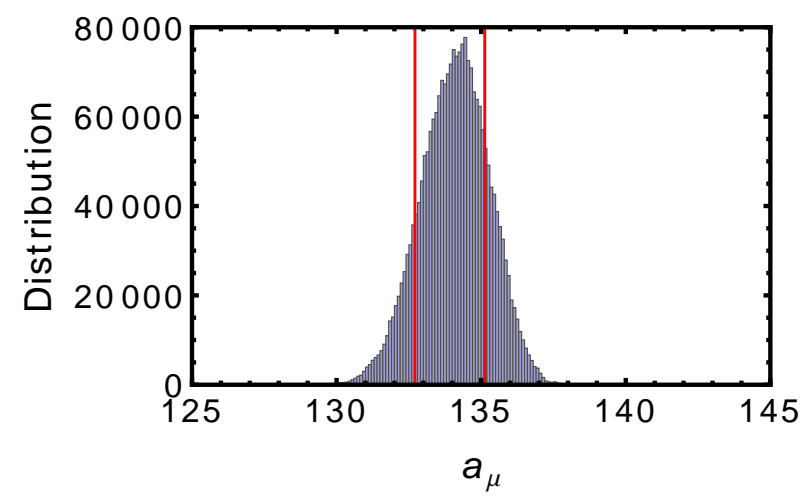

FIG. 3: Distribution of a typical $a_{\mu}$ sample obtained from the Monte Carlo simulation of pseudodata, with an input modulus measured by $B A B A R$ in the region $0.65-0.71 \mathrm{GeV}$. Details of the input are given in the text. The vertical lines delimitate the region of $68.3 \%$ C.L.

$0.65-0.71 \mathrm{GeV}$, shown in Fig. 11 The procedure was applied for each of the input phases, Bern and Madrid. The calculation was performed using as input each of the two spacelike values (9) and the best prediction was retained.

In Figs. 4 and 5 we show the $68.3 \%$ C.L. intervals of $a_{\mu}$ obtained from the Monte Carlo simulation described in the previous section, for all the timelike points used as input from the $e^{+} e^{-}$and $\tau$ experiments. The results have been obtained using as input the Bern phase [38, 39]. The Madrid phase leads to similar results. The bounds obtained with various values of the timelike modulus reflect the quality of data shown in Figs. 11 ranging between the most accurate, $B A B A R$, and those with the largest errors, OPAL.

We have then applied the averaging procedure described in the previous section, for combining the predictions available from different measurements of each experiment. The average was obtained using the robust prescription (13). For estimating the error, we have computed $\chi^{2}(f)$ defined in (14) and compared it with the number of degrees of freedom, $n-1$, where $n$ is the number of points in each panel of Figs. 4 and 5 It turned out that in all cases the ratio $\chi^{2}(0) /(n-1)$ was less than 1 and increased for a positive correlation, reaching unity for $f$ in general in the range $0.40-0.70$.

Some pathologies were encountered however in a few cases. One type of pathology is illustrated in Fig. 6. where we show the dependence on $f$ of the ratio $\chi^{2}(f) /(n-1)$ and of the standard deviation for the input from CMD2 and Madrid phase. In this case, the ratio becomes 1 only for values of $f$ close to 1 , where the variance $\sigma^{2}(f)$ calculated according to (16) starts to
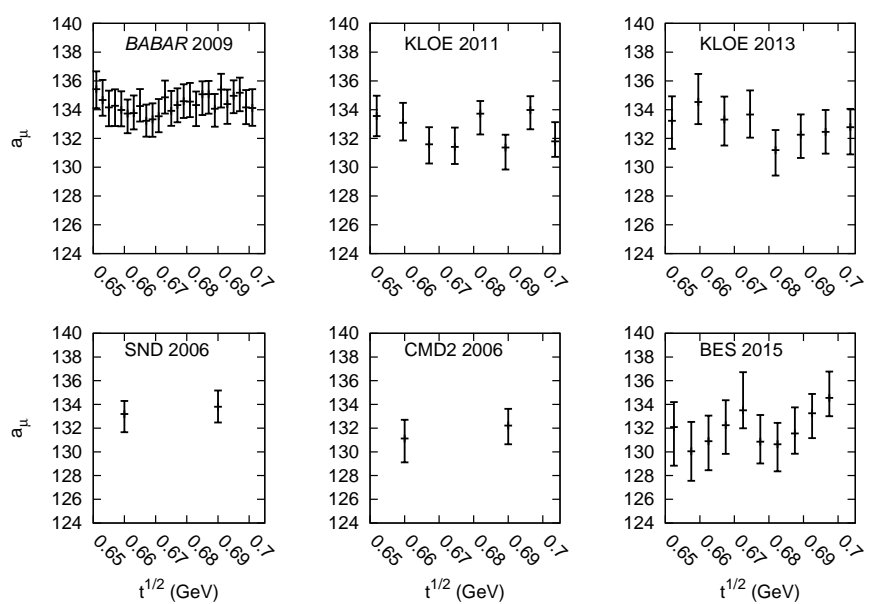

FIG. 4: Allowed intervals at $68.3 \%$ C.L. for the quantity $a_{\mu} \equiv$ $a_{\mu}^{\pi \pi(\gamma), \mathrm{LO}}\left[2 m_{\pi}, 0.63 \mathrm{GeV}\right] \times 10^{10}$, as a function of the energy in the region $(0.65-0.71) \mathrm{GeV}$ where the timelike modulus used as input was measured in $e^{+} e^{-}$experiments.
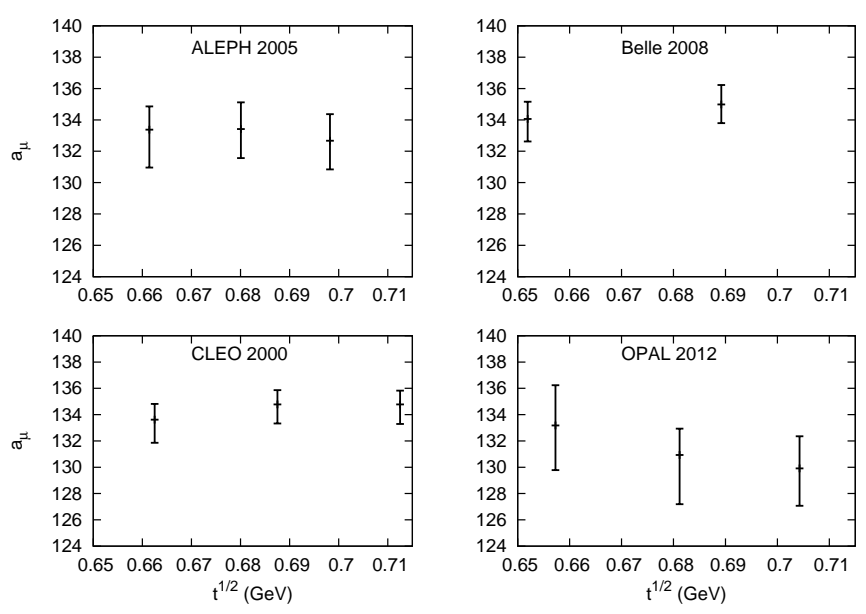

FIG. 5: Allowed intervals at $68.3 \%$ C.L. for the quantity $a_{\mu} \equiv$ $a_{\mu}^{\pi \pi(\gamma), \mathrm{LO}}\left[2 m_{\pi}, 0.63 \mathrm{GeV}\right] \times 10^{10}$, as a function of the energy in the region $0.65-0.71 \mathrm{GeV}$ where the timelike modulus used as input was measured in $\tau$ experiments.

decrease $^{2}$. This happens because the individual values are much closer than expected from the ascribed errors. As discussed in 45], in such cases the averaging cannot reduce the overall error, as the blind application of the

2 One can show that in all cases when the individual errors are different, $\sigma$ exhibits a decrease above a certain $f$ and vanishes for $f=1$. In the particular case of equal errors, the variance grows linearly with $f$, as discussed in [45]. 


\begin{tabular}{lcc}
\hline \hline & Bern phase & Madrid phase \\
\hline CMD2 06 & $131.804 \pm 1.563$ & $131.396 \pm 1.585$ \\
SND 06 & $133.535 \pm 1.371$ & $133.102 \pm 1.306$ \\
BABAR 09 & $134.338 \pm 0.939$ & $134.086 \pm 0.862$ \\
KLOE 11 & $132.560 \pm 1.220$ & $132.017 \pm 1.035$ \\
KLOE 13 & $132.864 \pm 1.413$ & $132.343 \pm 1.224$ \\
BESIII 15 & $131.958 \pm 1.725$ & $132.753 \pm 1.719$ \\
\hline CLEO 00 & $134.478 \pm 1.389$ & $133.897 \pm 1.183$ \\
ALEPH 05 & $133.114 \pm 1.703$ & $132.298 \pm 1.783$ \\
Belle 05 & $134.588 \pm 1.227$ & $134.280 \pm 1.136$ \\
OPAL 12 & $131.176 \pm 2.803$ & $129.910 \pm 2.970$ \\
\hline \hline
\end{tabular}

TABLE II: Central values and $68.3 \%$ C.L. standard deviations for the quantity $a_{\mu}^{\pi \pi(\gamma), \mathrm{LO}}\left[2 m_{\pi}, 0.63 \mathrm{GeV}\right] \times 10^{10}$, obtained by averaging the results shown in Figs. 4 and 5 for each experiment.

prescription would indicate. Therefore, for this case we adopt the modified prescription of taking the maximum variance for $f$ in the range $(0,1)$. The value of $\chi^{2}$ corresponding to this $f$ is smaller than 1 , which is due to the fact that the individual values to be averaged are very close. We encountered a similar situation with the data from ALEPH and both phases. In these cases, the combined error is not much less than the individual errors entering the combination.

A different type of pathology was encountered with KLOE 11 data: in this case, for both phases, the individual values are rather different and their errors are rather small. As a consequence, $\chi^{2}(f) /(n-1)$ becomes 1 for $f$ close to 0 . However, the corresponding variance (16) turns out to be much smaller than estimated from the spread of the individual values. Since these values are based on measurements of the modulus at different energies by the same experiment, the differences among them indicate a problem with the data and an error reduction by their combination is not reliable. Therefore, as a conservative error, we adopted in this case too the maximum variance for $f$ in the range $(0,1)$, whose magnitude is comparable with those of the individual errors. We illustrate this case in Fig. 7] where we show the dependence on $f$ of the ratio $\chi^{2}(f) /(n-1)$ and of the standard deviation for the input from KLOE 11 and Madrid phase.

Except these special cases, the standard deviation was calculated using (16), with the covariance matrix (15) corresponding to the fraction $f$ determined from the equation $\chi^{2}(f)=n-1$. A typical situation is shown in Fig. 8, where we show the dependence on $f$ of the ratio $\chi^{2}(f) /(n-1)$ and of the standard deviation for the input from BESIII and Madrid phase.

In Table II we present the results of the average procedure for all the $e^{+} e^{-}$and $\tau$ experiments. For completeness, we give the results obtained separately with the Bern and the Madrid phase.

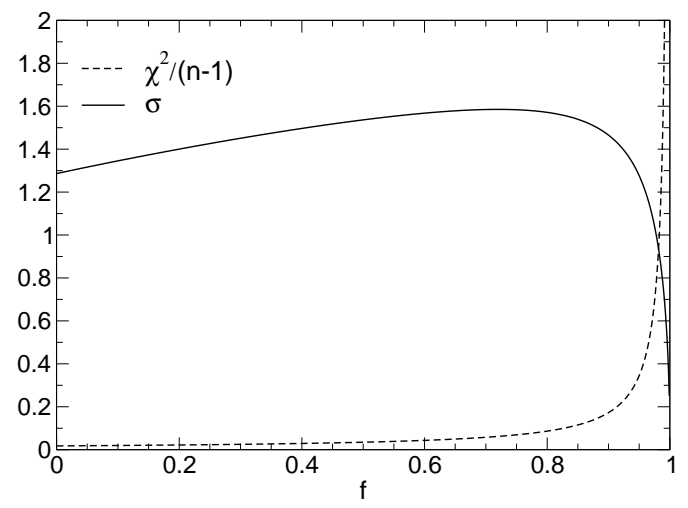

FIG. 6: Dependence on $f$ of the ratio $\chi^{2}(f) /(n-1)$ and the standard deviation, $\sigma \equiv \sqrt{\sigma^{2}(f)}$ for the timelike data measured by CMD2 and Madrid phase. The equality $\chi^{2}(f) /(n-$ $1)=1$ holds for large values of $f$.

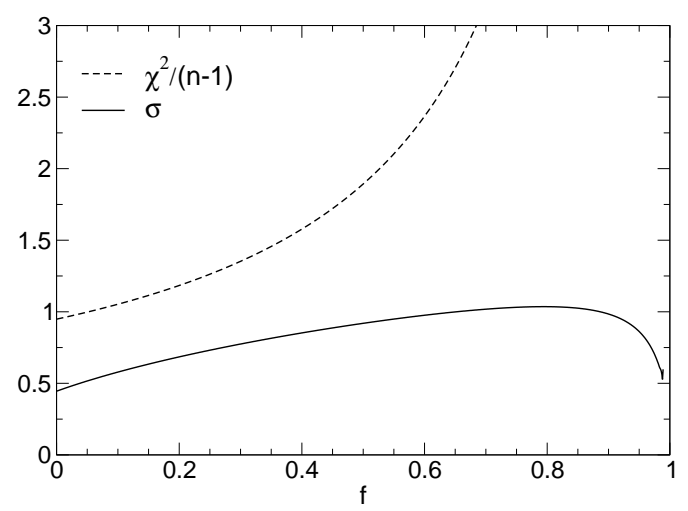

FIG. 7: Dependence on $f$ of the ratio $\chi^{2}(f) /(n-1)$ and the standard deviation, $\sigma \equiv \sqrt{\sigma^{2}(f)}$ for the timelike data measured by KLOE 11 and Madrid phase. The equality $\chi^{2}(f) /(n-1)=1$ holds for small values of $f$.

The last step is to combine the individual values obtained with measurements by different experiments. The correlation between these values is difficult to assess $a$ priori. There is of course a consistent common information going as input into all these determinations. However, the most important input, which has the crucial role in error reduction, is the modulus of the form factor in the region $0.65-0.71 \mathrm{GeV}$ measured by different experiments, which makes the difference between the values given in Table II. Some correlation might exist also between these measurements, but there is no consensus in the views on their treatment 33, 34. We therefore applied the same averaging procedure [45] suitable for cases when the correlations are not known.

The data from $e^{+} e^{-}$-annihilation and $\tau$-decay experiments are consistent in the region $0.65-0.71 \mathrm{GeV}$, so 


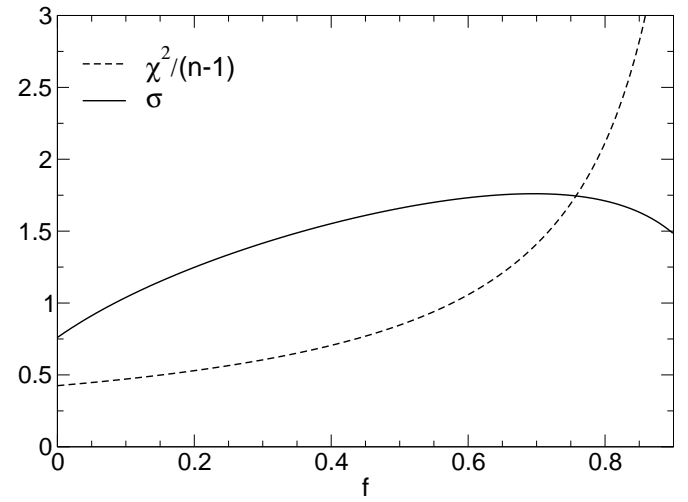

FIG. 8: Dependence on $f$ of the ratio $\chi^{2}(f) /(n-1)$ and the standard deviation, $\sigma \equiv \sqrt{\sigma^{2}(f)}$ for the timelike data measured by BESIII and Madrid phase. The error is obtained with $f$ determined from the equation $\chi^{2}(f) /(n-1)=1$.

the results from all the 10 experiments can be combined into a single average. The ratio $\chi^{2}(0) /(N-1)$, where $N=10$, turned out to be smaller than 1 , which indicates a positive correlation between the predictions of various experiments. By applying the prescription given in [45], the correlation was found to be $f=0.4$ for Bern phase and $f=0.3$ for Madrid phase, leading to the values of $a_{\mu}$ equal to $(133.425 \pm 0.793) \times 10^{-10}$ and $(133.092 \pm 0.653) \times 10^{-10}$, respectively ${ }^{3}$. Taking the simple average of the central values and errors obtained with the two phases, which are not statistically independent, we obtain the conservative final estimate ${ }^{4}$

$$
a_{\mu}^{\pi \pi(\gamma), \mathrm{LO}}\left[2 m_{\pi}, 0.63 \mathrm{GeV}\right]=(133.258 \pm 0.723) \times 10^{-10} .
$$

This result is consistent with our previous result reported in Ref. [35] and has a slightly smaller error. We emphasize that in 35] the prediction based on the present formalism was combined also with the direct integration of the cross section measured by $B A B A R$ at energies below $0.63 \mathrm{GeV}$, while in this work we do not use data from low energies.

\section{DISCUSSIONS AND CONCLUSION}

In this work, we have studied the two-pion contribution from energies below $0.63 \mathrm{GeV}$ to the muon $g-2$, by exploiting analyticity and unitarity of the pion pion electromagnetic form factor. The motivation of the work is

3 Assuming the values not correlated, one would obtain considerably smaller errors, 0.437 and 0.402 , respectively.

4 The separate combination of the results obtained with data from $e^{+} e^{-}$and $\tau$-decay experiments leads to the values $(133.018 \pm$ $0.766) \times 10^{-10}$ and $(133.785 \pm 0.993) \times 10^{-10}$, respectively. the relatively large error (of about $13.1 \times 10^{-11}$, see Ref. [32]) of this contribution obtained by direct data integration, explained by experimental difficulties in identifying pion pairs at low energies and the behavior of the QED kernel $K(t)$ in the integral (10) expressing $a_{\mu}$ in terms of the pion form factor modulus.

The main idea of our approach was to use, instead of the modulus, the phase of the pion form factor in the elastic region, equal by Fermi-Watson theorem to the phase shift of the $\mathrm{P}$-wave $\pi \pi$ amplitude, known with precision from the solution of Roy equations [38-40]. We have also used a conservative integral constraint on the modulus above the inelastic threshold, derived from $B A B A R$ data [16] and perturbative QCD [41 43], and two precise measurements of the form factor at spacelike values of the momentum transfer [53, 54].

A significant contribution to the final precision is brought by the inclusion in the input of several measurements of the modulus of the form factor at higher energies, from the $e^{+} e^{-}$-annihilation experiments CMD2, SND, $B A B A R$, KLOE 11, KLOE 13 and BESIII, and the $\tau$-decay experiments CLEO, ALEPH, OPAL and Belle. In practice we considered the energy region $0.65-0.71$ $\mathrm{GeV}$, where the modulus is measured with a better accuracy, and which is close enough to the low-energy region of interest such as to have a significant constraining power. From this input, using techniques of functional optimization theory, we derived rigorous constraints on the contribution to $a_{\mu}$ of the energies below $0.63 \mathrm{GeV}$, where the experimental data are poor. We emphasize that the formalism exploits in an optimal way the input information and requires no parametrization of the pion form factor. Furthermore, the results do not depend on the unknown phase of the pion form factor above the inelastic threshold.

The present analysis supersedes our previous work 35], where the same mathematical formalism was applied with data from only $4 e^{+} e^{-}$experiments (CMD2, SND, BABAR and KLOE 13). We included now as input data in the region $0.65-0.71 \mathrm{GeV}$ from 2 additional $e^{+} e^{-}$experiments, KLOE 2011 and BESIII 2016, and the measurements in the same region reported by $4 \tau$-decays experiments. The analysis has been also improved by a proper treatment with statistical tools of the uncertainties and the correlations between the input data. For each timelike input from the region $0.65-0.71 \mathrm{GeV}$ of a given experiment, we have evaluated a range for $a_{\mu}$ at a $68.3 \%$ confidence level. The results obtained with a definite input from the region $0.65-0.71 \mathrm{GeV}$ have been then combined using a statistical prescription suitable for cases when the correlations among the individual measurements are not precisely known [6, 45]. The combination of the values obtained with data from various experiments was done using the same prescription, which defines a robust central value and leads to a conservative error. By this procedure we have increased the reliability of our determination.

The final outcome of our analysis is expressed in Eq. 
(18). Our result is consistent with and more precise than the previous result reported in [35]. It has an uncertainty smaller by about $6 \times 10^{-11}$ than the direct integration of the cross section below $0.63 \mathrm{GeV} 32$.

Our work demonstrates that very general methods of unitarity and analyticity can be combined with high precision data from one sector to obtain stringent constraints in another sector. Using in addition suitable statistical methods to account for the uncertainties and the correlations of the input data, we obtained a significant improvement of the low-energy two-pion contribution to $a_{\mu}$. While the central value continues to remain stable, which in itself is a remarkable result, the fact that it has been possible to lower the uncertainty in this region by nearly a factor of two makes the results in this paper to be of significance. Until the accurate data expected from the CMD-3 and SND experiments at the VEPP-2000 $e^{+} e$ collider in Novosibirsk become available, our result represents the most precise and robust determination of the low-energy hadronic contribution to muon $g-2$.

\section{ACKNOWLEDGEMENTS}

The authors would like to dedicate this work to the loving memory of their friend and collaborator I. Sentitemsu Imsong who sadly passed away on October 30, 2015 while this work was in progress. We thank S. Eidelman, F. Jegerlehner, B. Malaescu, A. Nyffeler and M. Zhang for useful discussions in various stages of the work, and D. Boito for valuable information on the updated OPAL data. I.C. acknowledges support from CNCS-UEFISCDI, Contract Idei-PCE 121/2011 and from ANCS, Contract PN 164201 01/2016. The work of D.D. was supported by Deutsche Forschungsgemeinschaft Research Unit FOR 1873 Quark Flavour Physics and Effective Theories.

\section{Appendix A: SOLUTION OF THE EXTREMAL PROBLEM FORMULATED IN SEC. II]}

We must find optimal upper and lower bounds on $|F(t)|$ on the elastic unitarity cut, $t_{+}<t<t_{\text {in }}$ for $F(t) \in \mathcal{C}$, where $\mathcal{C}$ is the class of functions real analytic in the $t$-plane cut along the real axis for $t \geq t_{+}$, which satisfy the conditions 1-5 given in Sec. III. By means of a proof presented for the first time in Ref. [44], this problem can be reduced to a standard analytic interpolation problem [56] (also known as a Meiman problem [57]).

The first step of the proof is to define the Omnès function

$$
\mathcal{O}(t)=\exp \left(\frac{t}{\pi} \int_{4 m_{\pi}^{2}}^{\infty} d t^{\prime} \frac{\delta\left(t^{\prime}\right)}{t^{\prime}\left(t^{\prime}-t\right)}\right)
$$

where $\delta(t)$ is equal to $\delta_{1}^{1}(t)$ at $t \leq t_{\text {in }}$ and is an arbitrary smooth (Lipschitz continuous) function above $t_{\text {in }}$, which approaches asymptotically $\pi$.
It follows that the function $h(t)$ defined by

$$
F(t)=\mathcal{O}(t) h(t)
$$

is real on the real axis below $t_{\text {in }}$, therefore it is analytic in the $t$-plane cut only for $t>t_{\text {in }}$. In terms of $h(t)$, the equality (8) writes as

$$
\frac{1}{\pi} \int_{t_{\text {in }}}^{\infty} d t \rho(t)|\mathcal{O}(t)|^{2}|h(t)|^{2} \leq I .
$$

This relation can be written in a canonical form if we perform the conformal transformation

$$
\tilde{z}(t)=\frac{\sqrt{t_{\mathrm{in}}}-\sqrt{t_{\mathrm{in}}-t}}{\sqrt{t_{\mathrm{in}}}+\sqrt{t_{\mathrm{in}}-t}}
$$

and express the factors multiplying $|h(t)|^{2}$ in terms of an outer function, i.e. a function analytic and without zeros in the unit disk $|z|<1$. In practice, it is convenient to construct it as a product of two outer functions [44, 58]: the first one, denoted as $w(z)$, has the modulus equal to $\sqrt{\rho(t)|\mathrm{d} t / \mathrm{d} \tilde{z}(t)|}$. For the choice $\rho(t)=1 / t$, it is given by the simple expression

$$
w(z)=\sqrt{\frac{1-z}{1+z}} .
$$

The second outer function, denoted as $\omega(z)$, has the modulus equal to $|\mathcal{O}(t)|$, and can be calculated by the integral representation

$$
\omega(z)=\exp \left(\frac{\sqrt{t_{\text {in }}-\tilde{t}(z)}}{\pi} \int_{t_{\text {in }}}^{\infty} \frac{\ln \left|\mathcal{O}\left(t^{\prime}\right)\right| \mathrm{d} t^{\prime}}{\sqrt{t^{\prime}-t_{\text {in }}}\left(t^{\prime}-\tilde{t}(z)\right)}\right) .
$$

If we define the function $g(z)$ by

$$
g(z)=w(z) \omega(z) h(\tilde{t}(z))
$$

where $\tilde{t}(z)$ is the inverse of $z=\tilde{z}(t)$ defined in Eq. (A4), the condition (A3) can be written with no loss of information as

$$
\frac{1}{2 \pi} \int_{0}^{2 \pi} \mathrm{d} \theta|g(\zeta)|^{2} \leq I, \quad \zeta=e^{i \theta} .
$$

As shown in the analytic interpolation theory [56, 57], this condition leads to rigorous correlations among the values of the analytic function $g(z)$ and its derivatives at points inside the holomorphy domain, $|z|<1$. In particular, one can show (for a proof and earlier references see Ref. [58]) that (A8) implies the positivity condition

$$
\mathcal{D} \geq 0
$$

of the determinant $\mathcal{D}$ defined as

$$
\mathcal{D}=\left|\begin{array}{ccccc}
\bar{I} & \bar{\xi}_{1} & \bar{\xi}_{2} & \cdots & \bar{\xi}_{N} \\
\bar{\xi}_{1} & \frac{z_{1}^{2 K}}{1-z_{1}^{2}} & \frac{\left(z_{1} z_{2}\right)^{K}}{1-z_{1} z_{2}} & \cdots & \frac{\left(z_{1} z_{N}\right)^{K}}{1-z_{1} z_{N}} \\
\bar{\xi}_{2} & \frac{\left(z_{1} z_{2}\right)^{K}}{1-z_{1} z_{2}} & \frac{\left(z_{2}\right)^{2 K}}{1-z_{2}^{2}} & \cdots & \frac{\left(z_{2} z_{N}\right)^{K}}{1-z_{2} z_{N}} \\
\vdots & \vdots & \vdots & \vdots & \vdots \\
\bar{\xi}_{N} & \frac{\left(z_{1} z_{N}\right)^{K}}{1-z_{1} z_{N}} & \frac{\left(z_{2} z_{N}\right)^{K}}{1-z_{2} z_{N}} & \cdots & \frac{z_{N}^{2 K}}{1-z_{N}^{2}}
\end{array}\right|,
$$


in terms of the quantities

$$
\bar{I}=I-\sum_{k=0}^{K-1} g_{k}^{2}, \quad \bar{\xi}_{n}=\xi_{n}-\sum_{k=0}^{K-1} g_{k} z_{n}^{k} .
$$

where:

$$
\begin{aligned}
& g_{k}=\left[\frac{1}{k !} \frac{d^{k} g(z)}{d z^{k}}\right]_{z=0}, \quad 0 \leq k \leq K-1, \\
& \xi_{n}=g\left(z_{n}\right), \quad 1 \leq n \leq N .
\end{aligned}
$$

The inequality A9 defines an allowed domain for the real values $g\left(z_{n}\right)$ of the function at $N$ real points $z_{n} \in$ $(-1,1)$, and the first $K$ derivatives $g_{k}$ at $z=0$. In our application we consider $K=2$, noting that the coefficients $g_{0}$ and $g_{1}$ entering (A12) depend on the charge radius $\left\langle r_{\pi}^{2}\right\rangle$ defined in (7). We further take $N=3$, choosing two points as input, $t_{1}=t_{s}$ and $t_{2}=t_{t}$ from the conditions (9) and (10), while $t_{3}$ is an arbitrary point below $t_{\text {in }}$. For $t_{1}<0$ we have from Eqs. (A2) and A7)

$$
g\left(z_{1}\right)=w\left(z_{1}\right) \omega\left(z_{1}\right) F\left(t_{1}\right) / \mathcal{O}\left(t_{1}\right), \quad z_{1}=\tilde{z}\left(t_{1}\right) .
$$

while for $t_{n}, n=2,3$ we have

$$
g\left(z_{n}\right)=w\left(z_{n}\right) \omega\left(z_{n}\right)\left|F\left(t_{n}\right)\right| /\left|\mathcal{O}\left(t_{n}\right)\right|, \quad z_{n}=\tilde{z}\left(t_{n}\right),
$$

where the modulus $|\mathcal{O}(t)|$ of the Omnès function is obtained from (A1) by the principal value (PV) Cauchy integral

$$
|\mathcal{O}(t)|=\exp \left(\frac{t}{\pi} \mathrm{PV} \int_{4 m_{\pi}^{2}}^{\infty} d t^{\prime} \frac{\delta\left(t^{\prime}\right)}{t^{\prime}\left(t^{\prime}-t\right)}\right) .
$$

The condition (A9) provides the solution of the extremal problem formulated above: indeed, it can be written as a quadratic inequality for the unknown modulus $\left|F\left(t_{3}\right)\right|$. Recalling that $t_{3}$ is an arbitrary point in the elastic region, we obtain from (A9) the rigorous condition

$$
m \leq|F(t)| \leq M, \quad t<t_{\text {in }}
$$

where the bounds $m$ and $M$ are calculable in terms of known quantities.

One can prove [44, 58], that the bounds are optimal and their values do not depend on the unknown phase of the form factor above the inelastic threshold $t_{\text {in }}$. Furthermore, for a fixed weight $\rho(t)$ in (8), the bounds become stronger/weaker when the value of the r.h.s $I$ is decreased/increased, respectively. These properties make the formalism model independent and robust against the uncertainties from the high energy region.

In the procedure above we assumed only one input value on the modulus at a timelike energy. Actually, the general inequality (A9) allows the simultaneous inclusion of more input values, which are expected to lead to stronger bounds (A16) on the modulus below 0.63 $\mathrm{GeV}$. However, it turns out that when the number of input points is increased the procedure becomes quickly difficult numerically. It is more convenient to use as input only one modulus at a time and combine then the results taking into account the possible correlations between them.

\section{Appendix B: FORM FACTOR EXTRACTION FROM DATA}

In this appendix, we briefly discuss the extraction of the modulus of the form factor and the various corrections which must be taken into account while extracting the form factor from the data from $e^{+} e^{-}$annihilation and $\tau$ decay experiments. There is a vast literature on this subject (see [59]-64] and references therein).

In the case of $e^{+} e^{-}$experiments, the values of timelike form factor is extracted from the measured cross-section of $e^{+} e^{-} \rightarrow \pi^{+} \pi^{-}(\gamma)$. Several experimental collaborations (CMD2, SND, BABAR, KLOE) include the vacuum polarization (VP) into the definition of the pion form factor. Therefore, to obtain $|F(t)|$ we remove VP from the modulus quoted in Refs. [12, 15 17, 19, 20]. Equivalently, we use

$$
|F(t)|^{2}=\frac{3 t}{\alpha^{2} \pi \beta_{\pi}(t)^{3}} \frac{\sigma_{\pi \pi(\gamma)}^{0}(t)}{1+\frac{\alpha}{\pi} \eta_{\pi}(t)},
$$

where $\sigma_{\pi \pi(\gamma)}^{0}$ is the undressed cross-section obtained by removing VP and the $\rho-\omega$ interference factor from the measured cross-section, and $\eta_{\pi}(t)$ is the FSR factor discussed below Eq. (11).

The usefulness of $\tau$ decays for the calculation of the hadronic contribution to $a_{\mu}$ is based on conserved vector current (CVC) hypothesis, which implies the equality $F^{-}(t)=F(t)$ of the charged form factor $F^{-}(t)$ relevant in $\tau^{-} \rightarrow \pi^{-} \pi^{0} \nu_{\tau}$ decay and the form factor defined in (3). For a long time, $\tau$ hadronic decays offered the most precise data for the calculation of the hadronic contribution to $a_{\mu}$. The increasing precision of the $e^{+} e^{-}$experiments, in particular based on radiative return method, now make the two approaches comparable.

The $\tau$-decay data are given in terms of invariant hadronic mass squared distribution. The modulus of the pion form factor is extracted from the $\pi \pi$ distribution using the relation

$$
\begin{aligned}
\left|F^{-}(t)\right|^{2} & =\frac{2 m_{\tau}^{2}}{\left|V_{u d}\right|^{2}} \frac{1}{S_{E W}}\left(1-\frac{t}{m_{\tau}^{2}}\right)^{-2}\left(1+\frac{2 t}{m_{\tau}^{2}}\right)^{-1} \\
& \times \frac{\mathcal{B}_{\pi \pi}}{\mathcal{B}}\left(\frac{1}{N_{\pi \pi}} \frac{d N_{\pi \pi}}{d t}\right) \frac{1}{\beta_{-}^{3}(t)} \frac{1}{G_{E M}}
\end{aligned}
$$

where $\mathcal{B}_{\pi \pi}$ is the branching fraction for the $\tau$ decay into a dipion pair, $\mathcal{B}$ denotes the electron branching fraction, and $d N_{\pi \pi} / N_{\pi \pi} d t$ is the normalized invariant mass spectrum of the two-pion final state.

The expression (B2) includes all the corrections which ensure that $F^{-}(t)$ is the proper quantity to be used in the evaluation (11) of $a_{\mu}$. $S_{\mathrm{EW}}$ is a short distance correction to the effective four-fermion coupling $\tau^{-} \rightarrow \nu_{\tau}(d \bar{u})^{-}$ and $G_{\mathrm{EM}}$ is a long distance radiative correction involving real and virtual photons, calculated in [60] for the energy region of interest in our work. The isospin breaking due to the mass difference between charged and neutral pions 
is introduced through the phase-space

$\beta_{-}(t)=\left(1-\frac{\left(m_{\pi^{-}}+m_{\pi^{0}}\right)^{2}}{t}\right)^{1 / 2}\left(1-\frac{\left(m_{\pi^{-}}-m_{\pi^{0}}\right)^{2}}{t}\right)^{1 / 2}$,

relevant in $\tau$ decay. We have used standard values [6] for the masses and the CKM matrix element $\left|V_{\mathrm{ud}}\right|$. For $S_{\mathrm{EW}}$ we have used the values given by the experiments themselves.

Several other corrections considered in the literature are small and can be neglected in the region of interest, 0.65-0.71 GeV. The contribution due to the charged and neutral $\rho$ mass difference is negligible. The up and down quark mass difference accounting for charge-changing hadronic current between $u$ and $d$ quarks, which in turn leads to a breakdown of the CVC hypothesis, introduces a correction of the order of $\left(m_{u}-m_{d}\right)^{2} / m_{\tau}^{2} \simeq 10^{-5}$ 61] to $\mathcal{B}_{\pi \pi}$. Another correction suggested recently for $\tau$ data is produced by the $\rho-\gamma$ mixing [64]. The evaluation in a field-theoretic approach in 64 shows that the effect is important especially above the $\rho$ peak. We have studied the mixing thoroughly using the formulas given in [64] and did not find an appreciable effect at the lower energies we are interested in. We have therefore not included the effect.
[1] A. Czarnecki and W. J. Marciano, The muon anomalous magnetic moment: A harbinger for 'new physics', Phys. Rev. D 64, 013014 (2001), hep-ph/0102122.

[2] M. Davier and W. J. Marciano, The theoretical prediction for the muon anomalous magnetic moment, Ann. Rev. Nucl. Part. Sci. 54, 115 (2004).

[3] J. P. Miller, E. de Rafael and B. L. Roberts, Muon ( $g-$ 2): Experiment and theory, Rept. Prog. Phys. 70, 795 (2007), hep-ph/0703049.

[4] F. Jegerlehner and A. Nyffeler, The muon $g-2$, Phys. Rept. 477, 1 (2009), arXiv:0902.3360 [hep-ph]].

[5] J. P. Miller, E. de Rafael, B. L. Roberts and D. Stöckinger, Muon $(g-2)$ : Experiment and theory, Ann. Rev. Nucl. Part. Sci. 62, 237 (2012).

[6] K. A. Olive et al. [Particle Data Group Collaboration], Review of particle physics, Chin. Phys. C 38, 090001 (2014).

[7] C. M. Carloni Calame, M. Passera, L. Trentadue and G. Venanzoni, A new approach to evaluate the leading hadronic corrections to the muon $g$-2, Phys. Lett. B 746, 325 (2015), arXiv:1504.02228 [hep-ph]].

[8] G. Venanzoni [Muon g-2 Collaboration], The new muon $g-2$ experiment at Fermilab, Nucl. Phys. Proc. Suppl. 225-227, 277 (2012).

[9] T. Mibe [J-PARC g-2 Collaboration], Measurement of muon g-2 and EDM with an ultra-cold muon beam at J-PARC, Nucl. Phys. Proc. Suppl. 218, 242 (2011).

[10] G. W. Bennett et al. [Muon g-2 Collaboration], Final report of the muon E821 anomalous magnetic moment measurement at BNL, Phys. Rev. D 73, 072003 (2006), hep-ex/0602035.

[11] M. Benayoun et al., Hadronic contributions to the muon anomalous magnetic moment Workshop. $(g-2)_{\mu}$ : Quo Vadis Workshop. Mini proceedings, arXiv:1407.4021 [hep-ph].

[12] R. R. Akhmetshin et al. [CMD-2 Collaboration], Highstatistics measurement of the pion form factor in the rho-meson energy range with the CMD-2 detector, Phys. Lett. B 648, 28 (2007), hep-ex/0610021.

[13] R. R. Akhmetshin et al. [CMD-2 Collaboration], Reanalysis of hadronic cross-section measurements at CMD-2, Phys. Lett. B 578, 285 (2004). hep-ex/0308008.

[14] V. M. Aulchenko et al., Measurement of the $e^{+} e^{-} \rightarrow$ $\pi^{+} \pi^{-}$cross section with the CMD-2 detector in the 370 - 520-MeV c.m. energy range, JETP Lett. 84, 413
(2006) [Pisma Zh. Eksp. Teor. Fiz. 84, 491 (2006)], hep-ex/0610016.

[15] M. N. Achasov et al., Update of the $e^{+} e^{-} \rightarrow \pi^{+} \pi^{-}$ cross-section measured by SND detector in the energy region $400-\mathrm{MeV}<\sqrt{s}<1000-\mathrm{MeV}$, J. Exp. Theor. Phys. 103, 380 (2006) [Zh. Eksp. Teor. Fiz. 130, 437 (2006)], hep-ex/0605013.

[16] B. Aubert et al. [BaBar Collaboration], Precise measurement of the $e^{+} e^{-} \rightarrow \pi^{+} \pi^{-}(\gamma)$ Cross Section with the initial state radiation method at BABAR, Phys. Rev. Lett. 103, 231801 (2009), arXiv:0908.3589 [hep-ex]].

[17] J. P. Lees et al. [BaBar Collaboration], Precise measurement of the $e^{+} e^{-} \rightarrow \pi^{+} \pi^{-}(\gamma)$ cross section with the initial state radiation method at BABAR, Phys. Rev. D 86, 032013 (2012), arXiv:1205.2228 [hep-ex]].

[18] F. Ambrosino et al. [KLOE Collaboration], Measurement of $\sigma\left(e^{+} e^{-} \rightarrow \pi^{+} \pi^{-} \gamma(\gamma)\right)$ and the dipion contribution to the muon anomaly with the KLOE detector, Phys. Lett. B 670, 285 (2009), arXiv:0809.3950 [hep-ex]].

[19] F. Ambrosino et al. [KLOE Collaboration], Measurement of $\sigma\left(e^{+} e^{-} \rightarrow \pi^{+} \pi^{-}\right)$from threshold to $0.85 \mathrm{GeV}^{2}$ using initial state radiation with the KLOE detector, Phys. Lett. B 700, 102 (2011), arXiv:1006.5313 [hep-ex]].

[20] D. Babusci et al. [KLOE Collaboration], Precision measurement of $\sigma\left(e^{+} e^{-} \rightarrow \pi^{+} \pi^{-} \gamma\right) / \sigma\left(e^{+} e^{-} \rightarrow \mu^{+} \mu^{-} \gamma\right)$ and determination of the $\pi^{+} \pi^{-}$contribution to the muon anomaly with the KLOE detector, Phys. Lett. B 720, 336 (2013), arXiv:1212.4524 [hep-ex]].

[21] M. Ablikim et al. [BESIII Collaboration], Measurement of the $e^{+} e \rightarrow \pi^{+} \pi$ cross section between 600 and 900 $\mathrm{MeV}$ using initial state radiation, Phys. Lett. B 753, 629 (2016), arXiv:1507.08188 [hep-ex]].

[22] S. Anderson et al. [CLEO Collaboration], Hadronic structure in the decay $\tau^{-} \rightarrow \pi^{-} \pi^{0} \nu_{\tau}$, Phys. Rev. D 61, 112002 (2000), hep-ex/9910046.

[23] S. Schael et al. [ALEPH Collaboration], Branching ratios and spectral functions of $\tau$ decays: Final ALEPH measurements and physics implications, Phys. Rept. 421, 191 (2005), hep-ex/0506072.

[24] M. Davier, A. Höcker, B. Malaescu, C. Z. Yuan and Z. Zhang, Update of the ALEPH non-strange spectral functions from hadronic $\tau$ decays, Eur. Phys. J. C 74, no. 3, 2803 (2014), arXiv:1312.1501 [hep-ex]].

[25] K. Ackerstaff et al. [OPAL Collaboration], Measurement of the strong coupling constant alpha(s) and the vector 
and axial vector spectral functions in hadronic tau decays, Eur. Phys. J. C 7, 571 (1999), hep-ex/9808019.

[26] M. Fujikawa et al. [Belle Collaboration], High statistics study of the $\tau^{-} \rightarrow \pi^{-} \pi^{0} \nu_{\tau}$ decay, Phys. Rev. D 78, 072006 (2008), arXiv:0805.3773 [hep-ex]].

[27] M. N. Achasov et al., Hadronic cross section measurements at SND, Int. J. Mod. Phys. Conf. Ser. 35, 1460388 (2014).

[28] R. R. Akhmetshin et al., Current status of luminosity measurement with the CMD-3 detector at the VEPP$2000 e^{+} e^{-}$collider, JINST 9, C09003 (2014).

[29] V. M. Aulchenko et al. [SND Collaboration], Measurement of the $e^{+} e^{-} \rightarrow \eta \pi^{+} \pi^{-}$cross section in the centerof-mass energy range 1.22-2.00 GeV with the SND detector at the VEPP-2000 collider, Phys. Rev. D 91, no. 5, 052013 (2015), arXiv:1412.1971 [hep-ex]].

[30] G. V. Fedotovich et al., Preliminary results of measurements of hadronic cross sections with the CMD-3 detector at the VEPP-2000 electron-positron collider, Phys. Atom. Nucl. 78, no. 5, 591 (2015) [Yad. Fiz. 78, 635646 (2015)].

[31] S. Eidelman, Muon anomalous magnetic moment and hadronic vacuum polarization: Recent developments, PoS CD 15, 013 (2016).

[32] M. Davier, A. Höcker, B. Malaescu, C. Z. Yuan and Z. Zhang, Reevaluation of the hadronic contribution to the muon magnetic anomaly using new $e^{+} e^{-} \rightarrow \pi^{+} \pi^{-}$ cross section data from BABAR, Eur. Phys. J. C 66, 1 (2010), arXiv:0908.4300 [hep-ph]].

[33] M. Davier, A. Höcker, B. Malaescu and Z. Zhang, Reevaluation of the hadronic contributions to the muon $g-2$ and to $\alpha\left(M_{Z}\right)$, Eur. Phys. J. C 71, 1515 (2011), Erratum: [Eur. Phys. J. C 72, 1874 (2012)], arXiv:1010.4180 [hep-ph]].

[34] K. Hagiwara, R. Liao, A. D. Martin, D. Nomura and T. Teubner, $(g-2)_{\mu}$ and $\alpha\left(M_{Z}^{2}\right)$ re-evaluated using new precise data, J. Phys. G 38, 085003 (2011), arXiv:1105.3149 [hep-ph]].

[35] B. Ananthanarayan, I. Caprini, D. Das and I. S. Imsong, Two-pion low-energy contribution to the muon $g-2$ with improved precision from analyticity and unitarity, Phys. Rev. D 89, no. 3, 036007 (2014), arXiv:1312.5849 [hep$\mathrm{ph}]$.

[36] E. Fermi, Lectures on pions and nucleons, Nuovo Cim. 2S1, 17 (1955) [Riv. Nuovo Cim. 31, 1 (2008)].

[37] K. M. Watson, Some general relations between the photoproduction and scattering of $\pi$ mesons, Phys. Rev. 95, 228 (1954).

[38] B. Ananthanarayan, G. Colangelo, J. Gasser and H. Leutwyler, Roy equation analysis of $\pi \pi$ scattering, Phys. Rept. 353, 207 (2001), hep-ph/0005297.

[39] I. Caprini, G. Colangelo and H. Leutwyler, Regge analysis of the $\pi \pi$ scattering amplitude, Eur. Phys. J. C 72, 1860 (2012), arXiv:1111.7160 [hep-ph]].

[40] R. Garcia-Martin, R. Kaminski, J. R. Pelaez, J. Ruiz de Elvira and F. J. Yndurain, The pion-pion scattering amplitude. IV: Improved analysis with once subtracted Roy-like equations up to $1100 \mathrm{MeV}$, Phys. Rev. D 83, 074004 (2011), arXiv:1102.2183 [hep-ph]].

[41] G. R. Farrar and D. R. Jackson, The pion form factor, Phys. Rev. Lett. 43, 246 (1979).

[42] G. P. Lepage and S. J. Brodsky, Exclusive processes in quantum chromodynamics: Evolution equations for hadronic wave functions and the form factors of mesons,
Phys. Lett. B 87, 359 (1979).

[43] B. Melic, B. Nizic and K. Passek, Complete nextto-leading order perturbative QCD prediction for the pion form-factor, Phys. Rev. D 60, 074004 (1999) hep-ph/9802204.

[44] I. Caprini, Dispersive and chiral symmetry constraints on the light meson form-factors, Eur. Phys. J. C 13, 471 (2000), hep-ph/9907227.

[45] M. Schmelling, Averaging correlated data, Phys. Scripta 51, 676 (1995).

[46] H. Leutwyler, Electromagnetic form factor of the pion, hep-ph/0212324

[47] C. Hanhart, A new parameterization for the pion vector form factor, Phys. Lett. B 715, 170 (2012), arXiv:1203.6839 [hep-ph]].

[48] H. Czyz, A. Grzelinska, J. H. Kuhn and G. Rodrigo, The radiative return at $\Phi$ and $B$-factories: FSR for muon pair production at next-to-leading order, Eur. Phys. J. C 39, 411 (2005), hep-ph/0404078.

[49] Y. M. Bystritskiy, E. A. Kuraev, G. V. Fedotovich and F. V. Ignatov, The cross sections of the muons and charged pions pairs production at electron-positron annihilation near the threshold, Phys. Rev. D 72, 114019 (2005), hep-ph/0505236.

[50] B. Ananthanarayan, I. Caprini, D. Das and I. Sentitemsu Imsong, Parametrisation-free determination of the shape parameters for the pion electromagnetic form factor, Eur. Phys. J. C 73, 2520 (2013), arXiv:1302.6373 [hep-ph]].

[51] B. Ananthanarayan, I. Caprini and I. S. Imsong, Spacelike pion form factor from analytic continuation and the onset of perturbative QCD, Phys. Rev. D 85, 096006 (2012), arXiv:1203.5398 [hep-ph]].

[52] B. Ananthanarayan, I. Caprini, D. Das and I. S. Imsong, Model independent bounds on the modulus of the pion form factor on the unitarity cut below the $\omega \pi$ threshold, Eur. Phys. J. C 72, 2192 (2012), arXiv:1209.0379 [hep$\mathrm{ph}]]$.

[53] T. Horn et al. [Jefferson Lab F(pi)-2 Collaboration], Determination of the charged pion form factor at $Q^{2}=1.60$ and 2.45-(GeV/c $)^{2}$, Phys. Rev. Lett. 97, 192001 (2006), nucl-ex/0607005.

[54] G. M. Huber et al. [Jefferson Lab Collaboration], Charged pion form-factor between $Q^{2}=0.60-\mathrm{GeV}^{2}$ and $2.45-\mathrm{GeV}^{2}$. II. Determination of, and results for, the pion form-factor, Phys. Rev. C 78, 045203 (2008), arXiv:0809.3052 [nucl-ex]].

[55] D. Boito, M. Golterman, M. Jamin, A. Mahdavi, K. Maltman, J. Osborne and S. Peris, An updated determination of $\alpha_{s}$ from $\tau$ decays, Phys. Rev. D 85, 093015 (2012), arXiv:1203.3146 [hep-ph]].

[56] P. Duren, Theory of $H^{\mathrm{p}}$ Spaces, Academic Press, New York, 1970.

[57] N.N. Meiman, Analytic expressions for upper limits of coupling constants in quantum field theory, Sov. Phys. JETP 17, 830 (1963).

[58] G. Abbas, B. Ananthanarayan, I. Caprini, I. Sentitemsu Imsong and S. Ramanan, Theory of unitarity bounds and low energy form factors, Eur. Phys. J. A 45, 389 (2010), arXiv:1004.4257 [hep-ph]].

[59] M. Davier et al., The discrepancy between $\tau$ and $e^{+} e^{-}$ spectral functions revisited and the consequences for the muon magnetic anomaly, Eur. Phys. J. C 66, 127 (2010), arXiv:0906.5443 [hep-ph]].

[60] F. Flores-Baez, A. Flores-Tlalpa, G. Lopez Castro and 
G. Toledo Sanchez, Long-distance radiative corrections to the di-pion tau lepton decay, Phys. Rev. D 74, 071301 (2006), hep-ph/0608084.

[61] S. Tisserant and T. N. Truong, $\tau \rightarrow \delta \nu$ decay induced by light quark mass difference, Phys. Lett. B 115, 264 (1982).

[62] A. Pich, 'Anomalous' $\eta$ Production in tau decay, Phys. Lett. B 196, 561 (1987).
[63] H. Neufeld and H. Rupertsberger, Isospin breaking in chiral perturbation theory and the decays $\eta \rightarrow \pi l \nu$ and $\tau \rightarrow \eta \pi \nu$, Z. Phys. C 68, 91 (1995).

[64] F. Jegerlehner and R. Szafron, $\rho^{0}-\gamma$ mixing in the neutral channel pion form factor $F_{\pi}^{e}$ and its role in comparing $e^{+} e^{-}$with $\tau$ spectral functions, Eur. Phys. J. C 71, 1632 (2011), arXiv:1101.2872 [hep-ph]]. 